\title{
Synthesis of platinum nanoparticles with higher stability in ionic liquid-Nafion film and their electrocatalytic application
}

\author{
Qing Zhangl, Dawei Pan ${ }^{2,3}$, HaiYun Zhang ${ }^{2,3}$, HaiTao Han ${ }^{2,3,4}$ \\ ${ }^{1}$ The Key Laboratory in Molecular and Nano-materials Probes of the Ministry of Education of China, College of Chemistry, \\ Chemical Engineering and Materials Science, Shandong Normal University, Jinan, Shandong, 250014, People's Republic of \\ China \\ ${ }^{2}$ Key Laboratory of Coastal Environmental Processes and Ecological Remediation, Yantai Institute of Coastal Zone Research, \\ Chinese Academy of Sciences, Yantai, Shandong, 264003, People's Republic of China \\ ${ }^{3}$ Shandong Provincial Key Laboratory of Coastal Zone Environmental Processes, Yantai, Shandong, 264003, People's \\ Republic of China \\ ${ }^{4}$ School of Chemistry and Chemistry Engineering, Yantai University, Yantai, Shandong, 264005, People's Republic of China \\ E-mail:dwpan@yic.ac.cn
}

Published in Micro \& Nano Letters; Received on 28th April 2014; Revised on 24th December 2014; Accepted on 14th January 2015

\begin{abstract}
4-(3-methyl-1-imidazolio)-1-butanesulphonic acid triflate (BSMIMOTF)-Nafion film is introduced as a novel catalyst support. Nafion is used as the conductive membrane for BSMIMOTF immobilisation. A uniform distribution of spherical platinum nanoparticles (PtNPs) 10-30 nm in diameter can be electrochemically deposited on the membranous BSMIMOTF-Nafion matrix. Compared with the PtNPs on a bare and Nafionmodified electrode, the proposed sulphonyl-terminated ionic liquid (IL)-Nafion stabilised PtNPs exhibit higher electrocatalytic activity and better stability towards the electro-oxidation of methanol. The IL-Nafion film as a new catalyst support has great potential application in direct-methanol fuel cells.
\end{abstract}

1. Introduction: Direct-methanol fuel cells (DMFCs) are electro-chemical energy converters with great potential for use in various applications because of their simplicity, low pollution output and high efficiency [1-3]. However, the practical application of DMFCs is still limited by various issues, such as the low activity of anodic catalysts, continuous poisoning of the catalysts and the unwanted transference of methanol from anode to cathode. At present, the most active catalyst for methanol oxidation is platinum $(\mathrm{Pt})$. The electrocatalytic activity of $\mathrm{Pt}$ particles is affected by many factors, especially particle size and distribution [4-10]. The microenvironment significantly affects the morphology and electrocatalytic properties of nanoparticles (NPs) [11, 12].

Room temperature ionic liquids (RTILs) are ionic media resulting from the combination of organic cations and various anions. They exist in liquid state at room temperature and represent a new class of non-aqueous but polar solvents that can dissolve many compounds [13, 14]. Yang et al. [15] synthetised polyaniline/gold (Ag) nanocomposites using 1-butyl-3-methylimidazolium hexafluorophosphate, an RTIL. The synthesised polyaniline/Ag nanocomposites possess enhanced electrochemical activity and superior conductivity compared with the conducting polymers prepared by the conventional approach. Proteins such as enzymes also keep their activity and stability in RTILs better than in the conventional organic solvents or aqueous solutions [16]. RTILs have attracted considerable attention in electrochemistry because of their unique chemical and physical properties, such as high chemical and thermal stability, negligible vapour pressure and high conductivity [17, 18]. The high viscosity of ionic liquids (ILs) is necessary for their application as carbon paste electrode binders $[19,20]$. ILs have been widely used as electrolytes because of their wide electrochemical potential window [21]. For instance, Suryanto et al. [22] investigated the controlled electrodeposition of silver onto glassy carbon (GC), Ag and indium tin oxide-coated glass substrates from three room-temperature protic ILs. RTILs are also highly suitable media for the electrodeposition of metals [23]. Wang et al. [24] reported a novel formaldehyde (HCHO) sensor synthesised by the electrodeposition of AgPd alloy NPs on a chitosan-IL composite. Small-diameter AgPd NPs are well dispersed over the surface of the composite film, and the resulting electrode exhibits high electrocatalytic activity and stability in the electrooxidation of HCHO. In addition, Liu et al. [25] synthesised uniform CuPt NPs on IL-assisted graphene. However, the application of IL with functional groups acting as a support material for metal deposition has yet to be reported.

In the work reported in this Letter, we investigated a novel sulphonyl-terminated RTIL, 4-(3-methyl-1-imidazolio)-1-butanesulphonic acid triflate (BSMIMOTF), which was immobilised by Nafion, and pioneered the use of this RTIL as a catalyst support for metal deposition. As a hydrophilic RTIL, BSMIMOTF has interconnected ionic channels because of the exposed hydrophilic $\mathrm{SO}_{3}^{-}$, which is expected to alter the electrodeposition process to some degree. Uniform PtNPs were synthesised by electrochemical deposition on a BSMIMOTF-Nafion-modified electrode. The proposed PtNPs were analysed by scanning electron microscopy (SEM), energy-dispersive X-ray microanalysis (EDX) and electrochemistry. The electrocatalytic activity of PtNPs to methanol electro-oxidation was significantly enhanced by using BSMIMOTF as a new catalyst support.

\section{Experimental}

2.1. Chemicals: BSMIMOTF was supplied by the Shanghai Cheng Jie Chemical Co. Ltd. Nafion (5\%) was purchased from Sigma. All other chemicals were analytical reagents and used without further purification. All experiments were carried out at room temperature. Deionised water $(18.2 \mathrm{M} \Omega \cdot \mathrm{cm}$ specific resistance) obtained with a Pall Cascada Laboratory Water System was used in all experiments.

2.2. Apparatus and measurement: The morphology of the modified electrodes was characterised by SEM (Hitachi S-4800 microscope, Japan). The specimens were examined at magnifications of $20000 \times$ and $60000 \times$. The elemental compositions of the modified electrodes were characterised by EDX (HORIBA 
EX-350). All electrochemical experiments were carried out in a conventional three-electrode cell controlled by the CHI 660D Electrochemical Work Station (CH Instruments, Inc.). A modified GC disc ( $3 \mathrm{~mm}$ in diameter) was used as the working electrode, with $\mathrm{Ag} / \mathrm{AgCl}$ and $\mathrm{Pt}$ foil serving as the reference and counterelectrodes, respectively. Cyclic voltammogram (CV) and amperometric $i-t$ curve techniques were used for the electrochemical characterisation of the modified electrodes. The amperometric $i-t$ curve technique was also used for the electrodeposition of PtNPs.

2.3. Preparation of the modified electrode: Prior to use, a GC electrode was polished to a mirror over a microcloth with 0.05 $\mu \mathrm{m}$ alumina slurry and then rinsed and ultrasonicated by deionised water. BSMIMOTF $(15 \mu \mathrm{l})$ was dispersed in a solution of Nafion $(1 \%, 1.0 \mathrm{ml})$ using ultrasonic agitation to yield a uniform solution. Then, $4 \mu \mathrm{l}$ of the mixture solution was dropped on a clean GC electrode and dried at room temperature to form a uniformly coated electrode (BSMIMOTF-Nafion/GCE). Electrochemical deposition of $\mathrm{Pt}$ was performed in an aqueous solution of $1.93 \mathrm{mM} \mathrm{H} \mathrm{H}_{2} \mathrm{PtCl}_{6} \cdot 5 \mathrm{H}_{2} \mathrm{O}$. The electroplating potential was $-0.3 \mathrm{~V}$, with an electric charge quantity of $0.0045 \mathrm{C}$. The obtained PtNPs/BSMIMOTF-Nafion/GCE was washed carefully
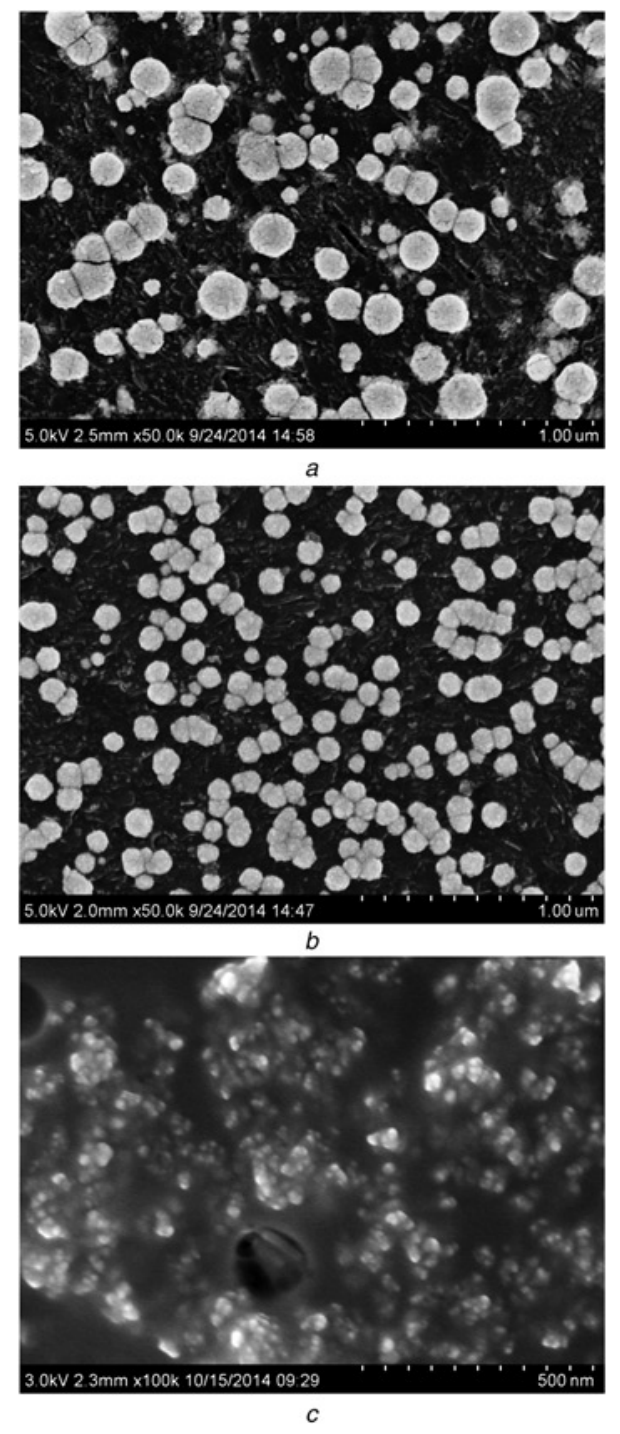

Figure 1 SEM images

$a$ PtNPs/GCE

$b$ PtNPs/Nafion/GCE

$c$ PtNPs/Nafion-IL/GCE with deionised water and then dried at room temperature. PtNPs/ GCE and PtNP/Nafion/GCE were fabricated using a similar method with the same deposition charges for comparison.

3. Results and discussion: Fig. $1 a$ illustrates that large Pt particles with diameters of 70-200 nm are dispersed on the GC electrode surface via direct electrochemical deposition. The smaller Pt particles with diameters of $50-100 \mathrm{~nm}$ can be observed on the Nafion-modified GC electrode (Fig. 1b). Besides the relative large particle size, the distributions of $\mathrm{Pt}$ particles on the bare and Nafion-modified GC surface are inhomogeneous. Comparatively, a uniform distribution of spherical PtNPs can be obtained by direct electrochemical deposition on BSMIMOTF-Nafion modified GCE (Fig. 1c). With an almost equal Pt deposition charge applied, the density of the Pt particles is apparently higher. This finding implies that BSMIMOTF-Nafion film modified GCE is a suitable support material for Pt deposition. In addition, the SEM image of the PtNPs/BSMIMOTF-Nafion/GCE in Fig. $1 c$ shows the membranous structure of the BSMIMOTFNafion-modified GCE. The particle size of the PtNPs ranges from 10 to $30 \mathrm{~nm}$, with uniform dispersion on the BSMIMOTF-Nafion matrix. This phenomenon is similar to the reported literature [26, 27]. ILs have low interfacial tension, which can improve the nucleation rate [26]. The polarity divergence has made BSMIMOTF a buffer passage to balance the phase difference, which is accordingly favourable for the transport of $\mathrm{PtCl}_{6}^{2-}$. Thus, a quasi-homogeneous distribution of $\mathrm{PtCl}_{6}^{2-}$ can be soundly achieved. As to the growth of PtNPs, it seems more difficult to initially drive $\mathrm{PtCl}_{6}^{2-}$ in water to migrate towards the surface of the BSMIMOTF-modified electrode, which results in less attachment of PtNPs yet with more uniform dispersion [27]. In addition, BSMIMOTF-Nafion has interconnected ionic channels because of the exposed hydrophilic $\mathrm{SO}_{3}^{-}$. Ion exchange occurs between $\mathrm{Pt}^{4+}$ and $\mathrm{SO}_{3}^{-}$upon dipping the BSMIMOTFNafion-modified GCE in electrolyte ions. Thus, the deposition of PtNPs on the BSMIMOTF-Nafion-modified electrode occurs in two ways: (a) part of the PtNPs is electrochemically deposited on the electrode directly from the aqueous solution of $\mathrm{H}_{2} \mathrm{PtCl}_{6} \cdot 5 \mathrm{H}_{2} \mathrm{O}$ and (b) another part of the PtNPs can be synthesised by the following reaction process

$$
\begin{gathered}
4\left[(\text { BSMIMOTF }- \text { Nafion }) \mathrm{SO}_{3}^{-}\right] / \mathrm{GCE}+\mathrm{Pt}^{4+} \stackrel{\text { ion-exchange }}{\longrightarrow} \\
{\left[(\mathrm{BSMIMOTF}-\mathrm{Nafion}) \mathrm{SO}_{3}\right]_{4} \mathrm{Pt} / \mathrm{GCE}} \\
{\left[(\text { BSMIMOTF }- \text { Nafion }) \mathrm{SO}_{3}\right]_{4} \mathrm{Pt} / \mathrm{GCE} \stackrel{\text { reduction }}{\longrightarrow}} \\
\text { PtNPs } / \text { BSMIMOTF }- \text { Nafion/GCE }
\end{gathered}
$$

The chemical composition of the obtained PtNPs/BSMIMOTFNafion/GCE composite was determined using EDX analysis (Fig. 2). The result shows that $\mathrm{C}, \mathrm{O}, \mathrm{F}, \mathrm{Pt}, \mathrm{Si}$ and $\mathrm{S}$ are the major elements. Si may have come from the silicone sealant. $\mathrm{F}$ and $\mathrm{O}$ come from the Nafion and the BSMIMOTF. S solely originates

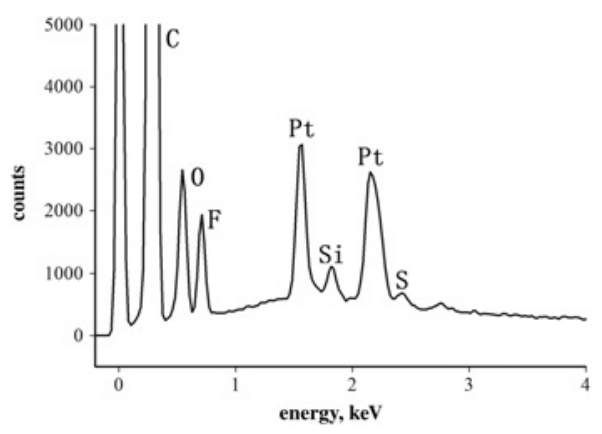

Figure 2 EDX spectrum of PtNPS/BSMIMOTF-Nafion/GCE 
Table 1 Results of the EDX analyses of the PtNPs/CS, PtNPs/Nafion/CS and PtNPs/BSMIMOTF-Nafion/CS

\begin{tabular}{lccc}
\hline Element & $\begin{array}{c}\text { PtNPs/CS, } \\
\text { wt } \%\end{array}$ & $\begin{array}{c}\text { PtNPs/Nafion/ } \\
\mathrm{CS}, \mathrm{wt} \%\end{array}$ & $\begin{array}{c}\text { PtNPs/BSMIMOTF-Nafion/ } \\
\mathrm{CS}, \mathrm{wt} \%\end{array}$ \\
\hline $\mathrm{C}$ & 12.22 & 15.84 & 22.23 \\
$\mathrm{O}$ & 14.80 & 14.46 & 18.44 \\
$\mathrm{Pt}$ & 72.98 & 37.44 & 32.54 \\
$\mathrm{~F}$ & & 32.26 & 14.57 \\
$\mathrm{~S}$ & & & 12.21 \\
\hline
\end{tabular}

from the BSMIMOTF. The existence of Pt demonstrates that the NPs inlaid in the BSMIMOTF-Nafion are actually Pt. In addition, the weight ratios of the Pt element in three matrices were studied in a copper sheet (CS) electrode and the corresponding results are shown in Table 1 by subtracting the copper ratio. The results have shown that the weight ratios of $\mathrm{Pt}$ are almost the same because of the same deposition charge. The existence of ILNafion film cannot prevent the deposition of Pt but results in smaller particles and a unique morphology.

Typical CVs for the PtNPs/BSMIMOTF-Nafion/GCE, BSMIMOTF-Nafion/GCE and bare GCE are between -0.2 and $1.0 \mathrm{~V}$ in a $0.5 \mathrm{M} \mathrm{H}_{2} \mathrm{SO}_{4}$ solution (Fig. 3). As shown in the inset, the BSMIMOTF-Nafion/GCE appears to conduct larger electric current than the bare GCE. BSMIMOTF has good ionic conductivity and can enhance the electrode's electron transfer rate. In addition, the membranous structure of BSMIMOTF-Nafion (Fig. 1c) increases the surface area of the electrode. These results imply that the BSMIMOTF-Nafion-modified GCE has better electrochemical performance than the bare GCE. In addition, the characteristic feature of $\mathrm{Pt}$ is displayed at the voltammogram of the PtNPs/BSMIMOTF-Nafion/GCE. Three distinctive $\mathrm{H}$ adsorptiondesorption peaks are detected at $-0.20,-0.17$ and -0.03 , followed by a double layer region corresponding to metallic Pt. With the potential positive shifting, the reduction peak of the $\mathrm{Pt}$ deposit is detected at $0.45 \mathrm{~V}$.

The electrocatalytic ability of the PtNPs/BSMIMOTF-Nafion/ GCE towards the electrochemical oxidation of methanol was investigated in $0.5 \mathrm{M} \mathrm{H}_{2} \mathrm{SO}_{4}$ solution containing $1 \mathrm{M} \mathrm{MeOH}$. As shown in Fig. 4, the features of the CV curve at the PtNPs/GCE and PtNPs/ Nafion/GCE are significantly similar to that at the PtNPs/ BSMIMOTF-Nafion/GCE. In particular, the potentials of the two peaks for methanol oxidation are almost the same at both electrodes, showing that methanol oxidation is not disturbed by the

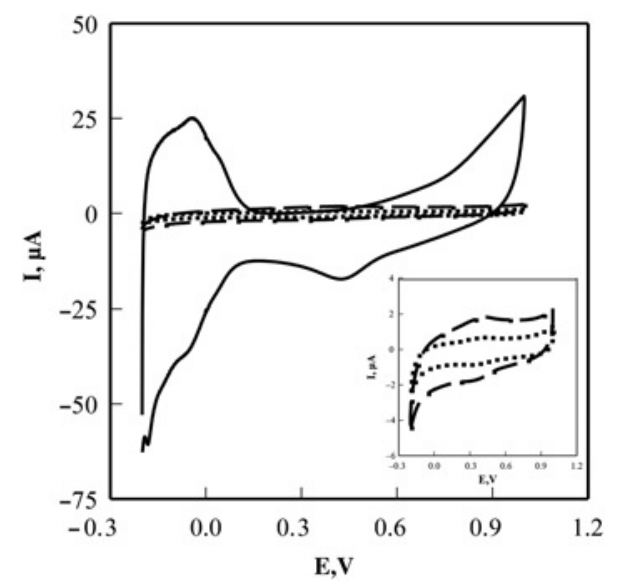

Figure $3 \mathrm{CVS}$ of PtNPS/BSMIMOTF-Nafion/GCE (solid line), BSMIMOTF-Nafion/GCE (dashed line) and bare GCE (dotted line) in $0.5 \mathrm{M} \mathrm{H}_{2} \mathrm{SO}_{4}$

Scan rate is $100 \mathrm{mV} / \mathrm{s}$

Inset: CVs of BSMIMOTF-Nafion/GCE and bare GCE

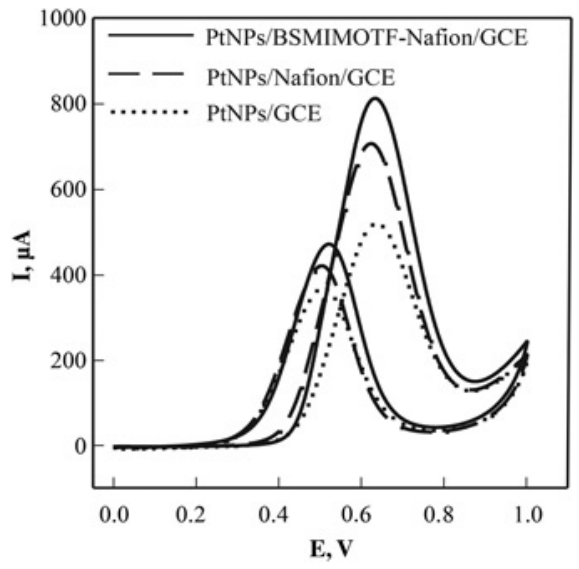

Figure $4 \mathrm{CV}$ s of PtNPs/BSMIMOTF-Nafion/GCE, PtNPs/Nafion/GCE and $\mathrm{PtNPs} / \mathrm{GCE}$ in $0.5 \mathrm{M} \mathrm{H}_{2} \mathrm{SO}_{4}+1 \mathrm{M} \mathrm{MeOH}$

Scan rate is $10 \mathrm{mV} / \mathrm{s}$

modification using BSMIMOTF. Two oxidation peaks of methanol can be observed clearly at 0.65 and $0.50 \mathrm{~V}$, respectively. The most negative peak of the oxidation of methanol at PtNPs/Nafion/GCE can be observed, which indicates that the PtNPs in the Nafion matrix are more active than that in the BSMIMOTF-Nafion matrix. A similar situation can also be observed at the PtNPs/IL/graphene electrode [27]. However, compared with the bare GCE, the PtNPs/BSMIMOTF-Nafion/GCE and the PtNP/Nafion/GCE show excellent catalytic properties towards methanol oxidation at the same deposition charge $(0.0045 \mathrm{C})$. Moreover, the PtNPs/ BSMIMOTF-Nafion/GCE exhibits a higher methanol oxidation current density than the PtNPs/Nafion/GCE. The small peak shifts of the oxidation of methanol might be caused by the unique surface properties of the electrodes.

The ratio of the forward anodic peak current density $\left(I_{\mathrm{F}}\right)$ to the reverse anodic peak current density $\left(I_{\mathrm{B}}\right)$ can be used to measure the tolerance of the catalyst to partially oxidised species accumulated on the surface of the electrode. A higher ratio indicates more effective removal of the poisoning species on the electrode surface [28]. The IF/IB ratios at the PtNPs/BSMIMOTF-Nafion/ GCE, PtNPs/Nafion/GCE and PtNP/GCE peaks are 1.71, 1.65 and 1.42, respectively. This result implies that the PtNPs/ BSMIMOTF-Nafion/GCE shows better anti-poisoning ability for methanol oxidation than the PtNPs/Nafion/GCE and PtNPs/GCE. The integration of PtNPs and BSMIMOTF in the Nafion polymeric matrix formed an electron-conducting (PtNPs) network and ion-conducting (BSMIMOTF and Nafion) matrix that improved the electronic and ionic transport capacity of the PtNPs/ BSMIMOTF-Nafion composite film, thereby causing a greater degree of oxidation completion and reducing poisoning. In addition, the efficiency may be attributed to the small particle size and high dispersion of PtNPs and the three-dimensional (3D) structure of the PtNPs/BSMIMOTF-Nafion.

The long-term stabilities of the PtNPs/BSMIMOTF-Nafion/ GCE, PtNPs/Nafion/GCE and PtNPs/GCE electrodes for methanol oxidation were also studied by chronoamperometric measurements. Fig. 5 shows typical $i-t$ curves at a constant potential of $0.6 \mathrm{~V}$ in a $0.5 \mathrm{M} \mathrm{H}_{2} \mathrm{SO}_{4}$ solution containing $1 \mathrm{M} \mathrm{MeOH}$. A slower decay of current density with time implies that the catalyst has good antipoisoning ability [29]. As shown in Fig. 5, the currents decrease rapidly during the early stage because of the formation of intermediate species. The oxidation current on the PtNPs/BSMIMOTFNafion/GCE is larger than that on the PtNPs/Nafion/GCE and $\mathrm{PtNP} / \mathrm{GCE}$ at all times. Electron-oxidation of methanol on PtNPs/ BSMIMOTF-Nafion/GCE possesses the highest initial current. Moreover, the decay of the oxidation current on the PtNPs/ BSMIMOTF-Nafion/GCE is the slowest. The current of the 


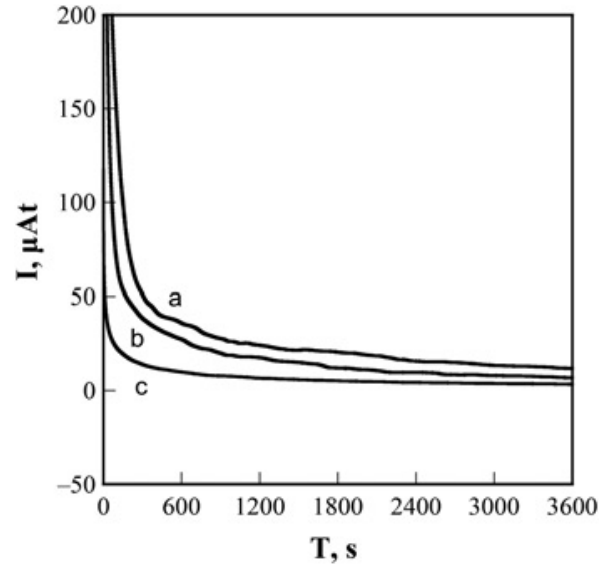

Figure 5 Amperometric $i-t$ curves of PtNP/BSMIMOTF-Nafion/GCE (curve a), PtNP/Nafion/GCE (curve b) and PtNP/GCE (curve c) for methanol oxidation in $0.5 \mathrm{M} \mathrm{H}_{2} \mathrm{SO}_{4}+1 \mathrm{M} \mathrm{MeOH}$

Fixed potential is $0.6 \mathrm{~V}$

PtNPs/BSMIMOTF-Nafion/GCE at $3600 \mathrm{~s}$ is 1.74 and 3.57 times greater than that of the PtNPs/Nafion/GCE and the PtNPs/GCE electrodes. Thus, the PtNPs on the BSMIMOTF-Nafion matrix have higher stability for methanol electro-oxidation because of the unique properties of IL-Nafion film.

4. Conclusion: This Letter illustrates the use of a BSMIMOTFNafion composite film as a support for the electrodeposition of PtNPs for methanol electro-oxidation. PtNPs with higher particle density and smaller particle diameter were dispersed on the BSMIMOTF-Nafion composite film because of the ion-exchange characteristics and excellent ionic conductivity of BSMIMOTFNafion. The PtNPs/BSMIMOTF-Nafion/GCE exhibits higher electrocatalytic activity for methanol oxidation than Pt/Nafion/ GCE and PtNP/GCE. This result can be attributed to the small particle size and high dispersion of $\mathrm{Pt}$, the unique properties of BSMIMOTF and the 3D structure of the modified electrode. These results show that sulphonyl-terminated IL as a new catalyst support has great potential application in DMFCs.

5. Acknowledgments: This work was financially supported by the National Natural Science Foundation of China (41276093 and 21275091), the Natural Science Foundation of Shandong Province (BS2010HZ030), the Taishan Scholar Program of Shandong Province and the Youth Innovation Promotion Association of the Chinese Academy of Science.

\section{References}

[1] Arico A.S., Creti P., Modica E., ET AL.: 'Investigation of direct methanol fuel cells based on unsupported Pt-Ru anode catalysts with different chemical properties', Electrochim. Acta, 2000, 45, (25), pp. $4319-4328$

[2] Ren X.M., Zelenay P., Thomas S., ET AL.: 'Recent advances in direct methanol fuel cells at Los Alamos National Laboratory', J. Power Sources, 2000, 86, (1), pp. 111-116

[3] Kang J., Wang R.F., Wang H., ET AL.: 'Effect of Ni core structure on the electrocatalytic activity of $\mathrm{Pt}-\mathrm{Ni} / \mathrm{C}$ in methanol oxidation', Materials, 2013, 6, (7), pp. 2689-2700

[4] Kohei U., Hideaki K., Yoshinobu N.: 'Structure of Pt microparticles dispersed electrochemically onto glassy carbon electrodes, examination with the scanning tunneling microscope and the scanning electron microscope', J. Electroanal .Chem., 1988, 256, (2), pp. 481-487

[5] Watanabe M., Saegusa S., Stonehart P.: 'High platinum electrocatalyst utilizations for direct methanol oxidation', J. Electroanal. Chem., 1989, 271, (1), pp. 213-220

[6] Tian N., Zhou Z.Y., Sun S.G., Ding Y., Wang Z.L.: 'Synthesis of tetrahexahedral platinum nanocrystals with high-index facets and high electro-oxidation activity', Science, 2007, 316, pp. 732-735
[7] Tian N., Zhou Z.Y., Sun S.G.: 'Platinum metal catalysts of high-index surfaces: from single-crystal planes to electrochemically shapecontrolled nanoparticles', J. Phys. Chem. C, 2008, 112, pp. 19801-19817

[8] Wei L., Fan Y.J., Wang H.H., Tian N., Zhou Z.Y., Sun S.G.: 'Electrochemically shape-controlled synthesis in deep eutectic solvents of Pt nanoflowers with enhanced activity for ethanol oxidation', Electrochim. Acta, 2012, 76, pp. 468-474

[9] Wei L., Fan Y.J., Tian N., ET AL.: 'Electrochemically shape-controlled synthesis in deep eutectic solvents - a new route to prepare Pt nanocrystals enclosed by high-index facets with high catalytic activity', J. Phys. Chem. C, 2012, 116, pp. 2040-2044

[10] Zhou Z.Y., Huang Z.Z., Chen D.J., Wang Q., Tian N., Sun S.G.: 'High-index faceted platinum nanocrystals supported on carbon black as high efficient catalysts for ethanol electrooxidation', Angew. Chem. Int. Ed., 2010, 49, pp. 411-414

[11] El-Deab M.S., Sotomura T., Ohsaka T.: 'Oxygen reduction at Au nanoparticles electrodeposited on different carbon substrates', Electrochim. Acta, 2006, 52, (4), pp. 1792-1798

[12] El-Deab M.S., Ohsaka T.: 'Electrocatalysis by design, effect of the loading level of Au nanoparticles-MnOx nanoparticles binary catalysts on the electrochemical reduction of molecular oxygen', Electrochim. Acta, 2007, 52, (5), pp. 2166-2174

[13] He M.Q., Sun K.Y., Xia J.X., ET AL.: 'Reactable ionic liquid-assisted solvothermal synthesis of flower-like bismuth oxybromide microspheres with highly visible-light photocatalytic performances', Micro Nano Lett., 2013, 8, (8), pp. 450-454

[14] Song X.C., Zheng Y.F., Ma R., ET AL.: 'Electrochemical polymerisation of poly(3-bromothiophene)/ $\mathrm{WO}_{3}$ nanocomposite films', Micro Nano Lett., 2011, 6, (4), pp. 273-276

[15] Yang W.R., Liu J.Q., Zheng R.K., ET AL.: 'Ionic liquid assisted synthesis of polyaniline/gold nanocomposite and its biocatalytic application', Nanoscale Res. Lett., 2008, 3, (11), pp. 468-472

[16] Rantwijk F.V., Sheldon R.A.: 'Biocatalysis in ionic liquids', Chem. Rev., 2007, 107, (6), pp. 2757-2785

[17] Welton T.: 'Room-temperature ionic liquids. Solvents for synthesis and catalysis', Chem. Rev., 1999, 99, (8), pp. 2071-2083

[18] Zhang Y., Cao H.M., Fei W.W., ET AL.: 'Direct electrochemistry and electrocatalysis of hemoglobin immobilized into halloysite nanotubes/room temperature ionic liquid composite film', Sens. Actuators, 2012, 162, (1), pp. 143-148

[19] Babaei A., Taheri A.R., Aminikhah M.: 'Nanomolar simultaneous determination of levodopa and serotonin at a novel carbon ionic liquid electrode modified with $\mathrm{Co}(\mathrm{OH})_{2}$ nanoparticles and multiwalled carbon nanotubes', Electrochim. Acta, 2013, 90, pp. 317-325

[20] Sun W., Wang X.Z., Zhu H.H., ET AL.: 'Graphene- $\mathrm{MnO}_{2}$ nanocomposite modified carbon ionic liquid electrode for the sensitive electrochemical detection of rutin', Sens. Actuators, 2013, 178, pp. 443-449

[21] Hapiot P., Lagrost C.: 'Electrochemical reactivity in room-temperature ionic liquids', Chem. Rev., 2008, 108, (7), pp. 2238-2264

[22] Suryanto B.H.R., Gunawan C.A., Lu X.Y., ET AL.: 'Tuning the electrodeposition parameters of silver to yield micro/nano structures from room temperature protic ionic liquids', Electrochim. Acta, 2012, 81, pp. $98-105$

[23] Xiao F., Zhao F.Q., Zhang Y.F., ET AL.: 'Ultrasonic electrodeposition of gold-platinum alloy nanoparticles on ionic liquid-chitosan composite film and their application in fabricating nonenzyme hydrogen peroxide', Sens. J. Phys. Chem. C, 2009, 113, (3), pp. 849-855

[24] Wang Q., Zheng J.B., Zhang H.F.: 'A novel formaldehyde sensor containing AgPd alloy nanoparticles electrodeposited on an ionic liquid-chitosan composite film', J. Electroanal. Chem., 2012, 674, pp. 1-6

[25] Liu Y.J., Huang Y.Q., Xie Y., ET AL.: 'Preparation of highly dispersed CuPt nanoparticles on ionic-liquid-assisted graphene sheets for direct methanol fuel cell', Chem. Eng. J., 2012, 197, pp. 80-87

[26] Rappathy D., Gopalan A.Y., Lee K.P.: 'Synergistic contributions of multiwall carbon nanotubes and gold nanoparticles in a chitosan-ionic liquid matrix towards improved performance for a glucose sensor', Electrochem. Commun., 2009, 11, (2), pp. 397-401

[27] Shi G.Y., Wang Z.H., Xia J.F., ET AL.: 'Mixed ionic liquids/graphemesupported platinum nanoparticles as an electrocatalyst for methanol oxidation', Electrochim. Acta, 2014, 142, pp. 167-172

[28] Li Y.J., Gao W., Ci L.J., ET AL.: 'Catalytic performance of Pt nanoparticles on reduced graphene oxide for methanol electro-oxidation', Carbon, 2010, 48, (4), pp. 1124-1130

[29] Zhang C.X., Hu J., Wang X.K., ET AL.: 'High performance of carbon nanowall supported Pt catalyst for methanol electro-oxidation', Carbon, 2012, 50, (10), pp. 3731-3738 